\title{
Left atrial strain and derived indexes in diagnosing of heart failure with preserved ejection fraction
}

\author{
Changsheng $\mathrm{Ma}^{1}$, Yuping Liao ${ }^{1}$, Jia $\mathrm{Li} \mathrm{Fan}^{1}$, Xin Zhao ${ }^{1}$, Bo $\mathrm{Su}^{1}$, and BingYuan Zhou ${ }^{1}$ \\ ${ }^{1}$ Affiliation not available
}

September 25, 2021

\begin{abstract}
Objectives. We sought to evaluate the ability of left atrial strain and derived index to discriminate patients with HFpEF from individuals with risk factors of HFpEF. Methods and results. A total of $n=389$ patients with risk factors for HFpEF finally was prospectively enrolled into the study, 51 of them were diagnosed with HFpEF by ESC diagnostic criteria. 55 patients were undergone left ventricular catheterization, 35 of them with LVEDP elevated. Left atrial strain was measured in all patients. Compared patients without HFpEF, LASr and LASr/(E/e') was lower in HFpEF; E/LASr, LAVi/LASr and LVMI/LASrwas higher in patients with HFpEF. After adjusted for hypertension, diabetes, chronic kidney disease, LVEF and NT-proBNP, multivariate logistic regression analyses showed that LASr and derived indexes(E/LASr, LASr/(E/e'), LAVi/LASr and LVMI/LASr) were still the predictors of HFpEF in their respective models. LASr had good diagnostic accuracy for HFpEF. Of the left atrial strain derived parameters, LVMI/LASr was the best discriminatory ability for HFpEF (AUC 0.796, cutoff value 5.2, specificity $82 \%$, sensitivity $73 \%$ ). LASr, LASr/(E/e'), LAVi/LASr and LVMI/LASr with higher AUC was superior to conventional echocardiographic measures of diagnosing HFpEF. LASr and derived indexes were incorporated into the ESC diagnostic criteria, LASr-HFA-PEF score system (AUC=0.804) had a higher detection rate of LVEDP[?]16mmHg than the HFA-PEF score system $(\mathrm{AUC}=0.781)$. Conclusion. LASr and derived indexes with good accuracy beyond conventional echocardiographic parameters discriminate HFpEF from patients with risk factors of HFpEF. LASr and derived indexes incorporated into the ESC diagnostic criteria will improve the diagnostic efficiency.
\end{abstract}

\section{Hosted file}

\begin\{CJK\}\{UTF8\}\{gbsn\}. \end\{CJK\}\selectlanguage\{english\}doc available at https://authorea. } com/users/437756/articles/539172-left-atrial-strain-and-derived-indexes-in-diagnosingof-heart-failure-with-preserved-ejection-fraction 